(Physiologisches Laboratorium in Bonn.)

\title{
Ueber den Einfluss des Tetanus der Muskeln auf die in ihm enthaltenen Säuren.
}

Von

\section{Dr. Joseph W, Warren.}

Diese Untersuchung stellt sich die Aufgabe, den Einfluss zu bestimmen, welchen der Tetanus der Muskeln auf die in Aether löslichen freien und gebundenen Säuren ausübt, wobei es sich wesentlich um die Milchsäuren handelt.

Die grosse Schwierigkeit dieser quantitativen Analysen liegt darin, dass verschiedene Milchsäuren im Muskel vorkommen, deren Zinksalze einen verschiedenen Gehalt an Krystallwasser darbieten. Bei der Wägung des Zinklactats hat man es also immer mit Gemengen zu thun. Eine Bestimmung des Zinkgehaltes dieser Gemenge gibt auch keinen ganz zuverlässigen Anhalt über die Menge der Milchsäure aus Gründen, die am Schlusse dieser Abhandlung erhellen werden.

Streng durchfühtrbar ist es aber, das "Säureaequivalent" des Muskels in Ruhe and Tetanus zu bestimmen, d. h. durch Titration zu ermitteln, ob die Zahl der sauren in Aether löslichen Molecüle zu oder abnimmt.

Herr Geheimrath Pflüg er schlug mir deshalb vor, mit Anwendung der gleich za beschreibenden Methode, die aus frischen Muskeln gewinnbaren in Aether löslichen Säuren mit den aus tetanisirten Muskeln stammenden quantitativ zu vergleichen. Bei diesen Versuchen hat er mich aufs freundlichste unterstitzt.

Die von mir erhaltenen Resultate habe ich vor einiger Zeit in meiner Inaugural-Dissertation mitgetheilt. Seitdem habe ich zwei wichtige Versuche angestellt, die das frither Gewonnene bestätigen und ergänzen. Bei der geringen Verbreitung, der die Dissertationen sich zu erfreuen pflegen, ist es vielleicht nicht un- 
erlaubt, den sachlichen Theil meiner kleinen Arbeit neben den neuen Versuchen hier zum Abdruck zu bringen. Ich habe alle Werthe noch einmal ausgerechnet und einige durch ein Missverständniss entstandene Fehler, die aber nichts an dem in der Dissertation mitgetheilten Verhältniss der Säuren ändern, beseitigt.

Da ich durch meine ärztliche Thätigkeit verhindert war die neuen Versuche früher auszuführen und somit meiner Arbeit diese neue Form zu geben, so ist es mir sehr erfreulich zu constatiren, dass eine ähnliche Untersuchung meine Resultate vollständig bestätigt hat. - Astaschewsky ${ }^{1}$ ) hat nämlich in Hoppe-Seyler's Laboratorium unter Anwendung einer andern Methode und mit Bestimmung der Milchsäure mittelst ihrer Zink- und Kalksalze ebenfalls eine Abnahme der Säuren im tetanisirten Muskel bewiesen. Berechnet man die Milchsäure als Schwefelsäure, so stimmen seine Zahlen zufriedenstellend mit den meinigen î̉berein. Da Astaschewsky wohl von meiner Dissertation, die im Juni 1880 erschien, also 5 volle Monate vor der Veröffentlichung. seiner Arbeit, sowie von ihrer Erwähnung in der neuen „Physiological Chemistry" Gamgee's (Bd. I. S. 360) keine Kenntniss hatte, so ist mir seine Bestätigung und Ergänzung meiner Angaben doppelt angenehm.

Die hier gebrauchte Methode (im Wesentlichen dieselbe, der sich Borszczow ${ }^{2}$ ) unter Scherers Leitung bediente) war folgende :

Das Thier wurde gleich nach dem Tode in Eis und Kochsalz verpackt, um hierin mehrere Stunden zu verbleiben. Nachdem der Thierleib durch und durch sehr kalt resp. fest gefroren war, wurde er enthäutet, das betreffende Fleisch abgetrennt, durch eine kleine Wurstmaschine zerkleinert, und in absoluten Alkohol gebracht. Später brachte ich das abgeschnittene Fleisch in Bechergläser, die in einer Kältemischung standen, um das Erweichen während dieser Behandlung thunlichst zu vermeiden. Nachdem das Fleisch etwa 24 Stunden unter häufigem Umrühren ansgezogen worden, wurde der Alkohol abgegossen und frischer

1) Ueber die Säurebildung u. d. Milchsäuregehalt der Muskeln. Zeitschrift für physiol. Chem. IV. Hft. 6. S. 397, herausgegeben am 4. Nov. 1880 .

2) Borszczow: Nachweisung der Milchsäure als normaler Bestandtheil der lebenden Muskelfasern, u. s. w. Inaug.-Diss. Würzburg 1861. 
nachgefuillt. Nach dem zweiten Auszug wurde das Fleisch mittelst eines Colirtuchs sorgfältig ausgedrückt und filtrirt. Man könnte vielleicht daran denken, dass bei einem derartigen Ausziehen das Fleisch die zu untersuchenden Substanzen nicht leicht oder nicht gleichmässig abgebe. Es ist wohl möglich, dass der tetanisirte Muskel für den Alkohol schwieriger zugänglich und in Folge dessen nicht vollständig ausgezogen werde. Bei einigen Versuchen wurde deshalb das Fleisch mit Alkohol zerrieben, ohne dass hierdurch eine andere Beschaffenheit des Auszuges bemerkbar wurde.

Die vereinigten alkoholischen Anszüge mit Schwefelsäure schwach angesäuert, wurden auf $70-80^{\circ}$ erwärmt, nm die eiweissartigen Körper zu fällen. Das Filtrat wurde mit Aetzbaryt alkalisch gemacht, der ïberschüssige Baryt durch Kohlensäure, die Kohlensäure durch Erwärmen entfernt. Vom Filtrat wurde der Alkohol abdestillirt und der Rest eingeengt. Nach Zusatz von Schwefelsäure wurde die Flüssigkeit mit einem grösseren Volum Aether ausgezogen. Das geschah so, dass etwa das 4-6fache Volum Aether mehrmals in Anwendung kam, da derselbe durch Abdestilliren immer wieder gewonnen wurde: somit wurde mit dem 16-20fachen Volum Aether ausgezogen. Der Aether wurde durch Destillation entfernt, der Rest mitWasser auf $200 \mathrm{ecm}$ verdiunnt und diese saure Flüssigkeit titrirt. Da ich dieses Volum bequem und zweckmässig fand, habe ich dasselbe als Schluss-Volum, worauf das letzte Extract in jedem Versuch gebracht wurde, beibehalten. Der Kürze halber werde ich den auf $200 \mathrm{cem}$ verdünnten Rest aus dem Aetherextract einfach Aetherauszug nennen.

Besonders lästig war das Ausziehen mit Aether. Die schön klare, gelbe Flüssigkeit schied sich beim Zusatz von Aether in zwei Schichten, in eine untere etwas trübe, gelbe und eine obere weissliche, schaumige, sehr klebrige Schichte, die der vollständigen Trennung der Aetherauszüge einige Schwierigkeiten machte. Es blieben nämlich leicht einige Tropfen dieser Schichte an dem Scheidetrichter zurück, wodurch mit Leichtigkeit etwas Schwefelsäure in den Aetherauszug gelangen konnte, wie es mir in dem ersten Versuch wirklich passirte. Auf diesen Uebelstand aufmerksam geworden, gelang es mir, die Trennung so auszuführen, dass in keinem Aetherauszug mehr Schwefelsäure vorhanden war. In dem ersten Versuch musste das Ausziehen wiederholt werden. 
Ein Hauptzweck dieser Methode ist natürlich jedwede Zersetzung durch Gährung oder Fäulniss zu verhindern. Ich glaube, dass dies durch die Verpackung in Eis, sowie durch Ausziehen mit absolutem Alkohol in zuverlässiger Weise zu erreichen ist. Ich will noch ausdrüicklich bemerken, dass ich bei jedem Versuch bestrebt war, die Verarbeitung der zu vergleichenden Portionen möglichst gleichmässig auszuführen. Der in Anwendung kommende Alkohol, sowie der Aether, wurde für die beiden Hälften eines jeden Versuches demselben Vorrath entnommen. Uebrigens wurde durch Benutzung entsprechender Quantitäten versucht, die Gleichmässigkeit noch vollständiger $\mathrm{zu}$ machen. Besonders hervorzaheben ist der Umstand, dass nur neutral reagirender Aether gebraucht wurde.

\section{Vorversuch I.}

Gährungsversuch mit Fleisch.

Um etwas von der gewöhnlichen Milchsäure zu gewinnen, verfuhr ich nach der bekannten Vorschrift von Bensch und erhielt reichliche Mengen schön krystallisirten milchsauren Zinks. Daneben probirte ich, ob eine ähnliche Mischung mit Fleisch, anstatt Milch und Käse (also Zucker, Weinsäure, Wasser, Fleisch und Zinkweiss), möglicherweise Paramilchsäure lieferte. Der Topf stand viele Tage unter günstigen Bedingungen. Das Auftreten von paramilchsaurem oder gewöhnlichem milchsaurem Zink wurde nicht beobachtet.

\section{Vorversuch II.}

Gährungsversuch mit Glykogen.

Um das Verhalten des Glykogens unter den Bedingungen der Milchsäuregährung zu studiren, wurde eine ähnliche Mischung mit Glykogen anstatt Zucker und Muskelbrei anstatt Käse bereitet und in den Brütofen gestellt. Das Glykogen stammte aus einer Ochsenleber und war nicht vollständig rein. Der Brütofen war mit einem Gasregulator versehen. Trotz möglicht günstiger Bedingungen war keine Milchsäuregährung zu constatiren.

Ich gehe nunmehr zu den Thierversuchen über.

\section{Versuch I.}

a) Drei mittelgrosse Kaninchen wurden Mittags geköpft, das Blut in Alkohol aufgefangen und die Leiber sogleich in die Kältemischung verpackt.

Dem einen entnahm ich einen Schenkel, um frisches Fleisch für den schon erwähnten Versuch zu erhalten. Nach etwa 7 Stunden betrug die Tem. peratur der Mischung $-13{ }^{\circ} \mathrm{C}$. Die steinharten Leiber wurden möglichst 
rasch enthäutet und das Fleisch der Schenkel und des Rückens abgetrennt, gewogen und in Alkohol gebracht. Hier verarbeitete ich Fleisch und Blut nach der angegebenen Methode zusammen. Als der Rest aus dem aetherischen Auszug schon auf $200 \mathrm{ccm}$ verdünnt worden und ich im Begriff war, die Titrirung auszuführen, prüfte ich auf $\mathrm{H}_{2} \mathrm{SO}_{4}$. Wie oben erwähnt, war etwas in die Lösung mit übergegangen, so dass ich genöthigt war, die noch rettbaren $170 \mathrm{ccm}$ dieses Aetherauszuges nochmals auszuziehen und den so gewonnenen Auszug zur Titrirung zu gebrauchen.

Das Gewicht des Fleisches betrug 697,0 gr. Als Mittel aus vier gut übereinstimmenden Bestimmungen erhielt jch den Werth 1 cem des Aetherauszuges $=0,738 \mathrm{~cm}$ einer gewissen Kalilauge. $1 \mathrm{ccm}$ dieser KOH-Lösung $=0,004793 \mathrm{gr} \mathrm{H}_{2} \mathrm{SO}_{4}$. Um den Werth für $1 \mathrm{gr}$ Fleisch zu erhalten hat man folgende Rechnung auszuführen:

$$
\begin{aligned}
1 \mathrm{gr} \text { Fleisch } & =0,738 \times 200 \times \frac{200}{170} \times \frac{1}{697}=0,249 \mathrm{ccm} \text { KOH-Lösung. } \\
& =0,0011935 \mathrm{gr} \mathrm{H}_{2} \mathrm{SO}_{4} .
\end{aligned}
$$

b) Einige Tage später wurden ebenfalls drei Kaninchen zu einem Tetanisirversuche verwendet. Diese waren aus demselben Stall wie die drei andern, worin sämmtliche Thiere sich seit längerer Zeit in denselben Verhältnissen befunden hatten.

Vom Rückenmark aus wurden besonders die Hinterläufe und die Rückenmuskeln in Thätigkeit gesetzt. Der Tetanus war sehr vollständig, nur leider so anhaltend, dass die Versuchsthiere nach nur 5/4 Stunden verendeten. Die weitere Behandlung war wie bei den andern. Nach 4 Stunden stand das Thermometer in der Kältemischung auf $-17^{\circ} \mathrm{C}$. Durch sorgfältigere Trennung gelang es mir hier wie in allen späteren Versuchen einen Uebergang von $\mathrm{H}_{2} \mathrm{SO}_{4}$ in den Aetherauszug zu vermeiden.

Gewicht des gewonnenen Fleisches $=970,2 \mathrm{gr}$.

Als Mittel aus drei Bestimmungen:

$1 \mathrm{ccm}$ des Aetherauszuges

$=0,7821 \mathrm{ccm}$ der obigen $\mathrm{KOH}-\mathrm{Lösung}$.

$$
\begin{aligned}
1 \mathrm{gr} \text { Fleisch } & =0,7821 \times 200 \times \frac{1}{970,2} \\
& =0,1612 \mathrm{ccm} \mathrm{KOH}-\mathrm{Lösung} . \\
& =0,0007726 \mathrm{gr} \mathrm{H}_{2} \mathrm{SO}_{4} .
\end{aligned}
$$

Vergleicht man diese Zahlen, so erkennt man, dass das tetanisirte Fleisch weniger Säuren geliefert hat als das der ruhenden Muskeln. Das Verbältniss kann man folgendermassen ausdrücken:

$$
\begin{gathered}
\mathrm{I}^{\mathrm{a}} \text { (ohne Tetanus): } \mathrm{I}^{\mathrm{b}} \text { (Tetanus) } \\
=0,0011935: 0,0007726 \\
=1: 0,647
\end{gathered}
$$


Hier ist der Werth der Säuren des nichttetanisirten Fleisches wahrscheinlich zu klein ausgefallen, da durch die Wiederholung des Ausziehens, wobei nur eine Portion des ursprïnglichen Auszuges gebrancht werden konnte, etwas von diesen Substanzen verloren ging.

\section{Versuch II.}

a) Zwei kleine Kaninchen wurden geköpft, ausbluten lassen und gleich in die Kältemischung gebracht. Nach einigen Stunden stand das Thermometer auf $-12^{\circ} \mathrm{C}$. Verarbeitung wie im Versuch I, mit der Abänderung, dass das Blut nicht mit verarbeitet wurde.

Gewicht des Fleisches $=500,5 \mathrm{gr}$.

$1 \mathrm{ccm}$ des Aetherauszuges

$=1,0885 \mathrm{ccm}$ einer andern KOH-Lösung.

$1 \mathrm{ccm}$ dieser KOH-Lösung $=0,004773 \mathrm{gr} \mathrm{H}_{2} \mathrm{SO}_{4}$.

$1 \mathrm{gr}$ Fleisch $=1,0885 \times 200 \times \frac{1}{500,5}=0,43496 \mathrm{ccm} \mathrm{KOH-Lösung}$

$$
=0,002076 \mathrm{gr} \mathrm{H}_{2} \mathrm{SO}_{4} \text {. }
$$

b) An demselben Tag wurden zwei Kaninchen wie im vorigen Versuch tetanisirt. Es waren ebenfalls 4 Thiere, welche sich längere Zeit in demselben Stall befunden hatten. Der Tetanus war sehr vollständig. Das eine Kaninchen stärb nach $3^{1 / 4}$ Stunden, das andere etwa eine Stunde später. Jedes wurde gleich nach seinem Tode geköpft und genau so verpackt wie die zwei andern nicht tetanisirten. Bei der Verarbeitung ist leider etwas von dem Alkoholauszug durch Verspritzen verloren gegangen, so dass der Vergleich unvollständig ist.

Gewicht des Fleisches $=646,0 \mathrm{gr}$.

$1 \mathrm{ccm}$ des Aetherauszuges

$=0,23742 \mathrm{ccm}$ derselben KOH-Lösung.

$1 \mathrm{gr}$ Fleisch $=0,23742 \times 200 \times \frac{1}{646}$

$=0,0735 \mathrm{ccm}$ KOH-Lösung.

$=0,0003508+x$ gr H$_{2} \mathrm{SO}_{4}$.

Ich schätzte den Verlust auf höchstens $1 / 5$. Setzt man ihn $=1 / 2$ um den Fehler möglichst auszugleichen, so hat man für diesen Versuch:

$1 \mathrm{gr}$ Fleisch $=0,0007016$ gr $\mathrm{H}_{2} \mathrm{SO}_{4}$.

Hier ist also unter allen Umständen eine bedeutende Abnahme der Säuren zu constatiren. Das Verhältniss ist:

$$
\begin{gathered}
\mathrm{II}^{\mathrm{a}} \text { (ruhend) }: \mathrm{II}^{\mathrm{b}} \text { (tetanisirt) } \\
=0,002076: 0,0007016 \\
=1: 0,3379
\end{gathered}
$$


Ueber d. Einfluss d. Tetanus d. Muskeln auf d. in ihm enthaltenen Säuren. 397

\section{Versuch III.}

Um möglichst gleiche Verhältnisse herzustellen, wurde nun beschlossen einen Schenkel eines Kaninchens zu lähmen, während der andere zum Tetanus gebraucht wurde. $\mathrm{Zu}$ diesem Ende wurden an einem kräftigen Thier die zugänglichen Nerven (Ischiadicus und. Cruralis) des linken Schenkels durchschnitten. Die Blutung war sehr gering. Nacb 48 Stunden wurde der gesunde Schenkel theils vom Rückenmark, theils von den Muskelansätzen aus tetanisirt. Der Versuch gelang sehr gut, nur wenige Muskeln des gelähmten Schenkels arbeiteten mit. Nach $4 \frac{1}{2}$ Stunden wurde das Thier getödtet und wie vorher (nur nicht geköpft) verpackt. Nach $41_{4}^{1}$ Stunden war die Temperatur $-20^{\circ} \mathrm{C}$. Das Fleisch des nicht tetanisirten Schenkels war steinhart, das des tetanisirten weniger fest gefroren. Zerkleinerung und weitere Behandlung wie oben.

a) Links. Gelähmter Schenkel. Gewicht des Fleisches $=119,0 \mathrm{gr}$.

$1 \mathrm{ccm}$ des Aetherauszuges

$=0,3233 \mathrm{ccm}$ einer verdünnten $\mathrm{NaOH}$-Lösung.

$1 \mathrm{com}$ dieser $\mathrm{NaOH}-L$ ösung $=0,001904 \mathrm{gr} \mathrm{SO}_{4} \mathrm{H}_{2}$.

Der Werth der Lauge war durch eine bekannte Lösung von $\mathrm{ClH}$, diese Lösung von $\mathrm{ClH}$ durch $\mathrm{NO}_{3} \mathrm{Ag}$-Lösung und diese letztere mit einer durch Wägung chemisch reinen $\mathrm{ClNa}$ bereiteten Lösung festgestellt.

$$
\begin{aligned}
1 \mathrm{gr} \text { Fleisch } & =0,3233 \times 200 \times \frac{1}{119} \\
& =0,543 \mathrm{ccm} \mathrm{NaOH}-\mathrm{Lösung} \\
& =0,001034 \mathrm{gr} \mathrm{H} \mathrm{H}_{4} .
\end{aligned}
$$

b) Rechts. Tetanus. Gewicht des Fleisches $=139,3 \mathrm{gr}$.

$1 \mathrm{ccm}$ des Aetherauszuges

$$
=0,2075 \mathrm{ccm} \text { NaOH-Lösung. }
$$

$1 \mathrm{gr}$ Fleisch $=0,2075 \times 200 \times \frac{1}{139.3}$

$$
\begin{aligned}
& =0,2979 \mathrm{ccm} \text { NaOH-Lösung } \\
& =0,000567 \mathrm{gr} \mathrm{H}_{2} \mathrm{SO}_{4} \text {. }
\end{aligned}
$$

Ein Vergleich dieser Zahlen ergibt folgendes Verhältniss:

$\mathrm{III}^{\mathrm{a}}$ (gelähmt) : $\mathrm{II}^{\mathrm{b}}$ (tetanisirt)

$$
\begin{gathered}
=0,001034: 0,000567 \\
=1: 0,548
\end{gathered}
$$

Also eine Abnahme beim Tetanus des einen Beins bei gleichzeitiger Lähmung des andern.

Gegen diesen Versuch kann man einwenden, dass durch die 48stündige Ruhe des gelähmten Beines Umsetzungen bedingt wurden, die eine wirkliche Vermehrung der Säuren durch den Tetanus verdeckten. Um auch dieses Verhältniss zu prïfen, wieder- 
holte ich den Versuch so, dass ich dem operirten Thier nur wenige Stunden Ruhe liess, um sich zu erholen.

\section{Versuch IV.}

Durchtrennung der Nerven des linken Beines eines mittelgrossen Kaninchens. An demselben Tag, etwa $2^{1 / 2}$ Stunden nach der Durchschneidung, Tetanisirung des gesunden Schenkels mit einem Pol auf dem Rückenmark und dem andern in den Muskelansätzen des Unterschenkels. Etwas unvollständiger Tetanus. Zeitweise arbeiteten viele Muskeln des gelähmten Schenkels mit. Nach 41/4 Stunden, da die Muskeln des betreffenden Beines ersehöpft waren, wurde das Thier durch Schlag auf den Nacken getödtet und ohne Blutverlust verpackt.

a) Links. Lähmung. Fleisch $=119,5 \mathrm{gr}$.

$1 \mathrm{ccm}$ des Aetherauszuges

$=0,2294$ der obigen NaOH-Lösung.

$1 \mathrm{gr}$ Fleisch $=0,2294 \times 200 \times \frac{1}{119,5}$

$=0,3839 \mathrm{ccm} \mathrm{NaOH}-\mathrm{Lösung}$.

$=0,000731 \mathrm{gr}^{\mathrm{H}} \mathrm{SO}_{4}$.

b) Rechts. Tetanus. Fleisch $=121,3 \mathrm{gr}$.

$1 \mathrm{ccm}$ des Aetheranszuges

$$
=0,1488 \mathrm{ccm} \text { NaOH-Lösung. }
$$

$1 \mathrm{gr}$ Fleisch $=0,1488 \times 200 \times \frac{1}{121,3}$

$$
\begin{aligned}
& =0,2453 \mathrm{ccm} \mathrm{NaOH} \text {-Lösung. } \\
& =0,000467 \mathrm{gr} \mathrm{H}_{2} \mathrm{SO}_{4} \text {. }
\end{aligned}
$$

Vergleicht man diese Zahlen, so sieht man, dass die 48stündige Dawer der Lähmung allein den gefundenen Unterschied nicht bedingt hatte. Dass in dem gelähmten Schenkel soviel weniger Säure gefunden wurde als in dem vorigen Versuche, hat vielleicht darin seinen Grund, dass diese Muskeln, wie oben erwähnt, doch zeitweilig mit arbeiteten.

Das Verhältniss für diesen Versuch ist:

$$
\begin{gathered}
\text { IV }^{\mathrm{a}} \text { (Lähmung) : IVb (Tetanus) } \\
=0,000731: 0,000467 \\
=1: 0,639
\end{gathered}
$$

Gegen die beiden letzten Versuche kann ferner der Einwand gemacht werden, dass der Tetanus von den Muskelansätzen aus elektrolytische Umsetzungen eingeleitet hatte, die die Abnahme der Säuren bedingte. Um auch diesen Einwand zu entkräften, wurde der Versuch noch einmal wiederholt. 
Ueber d. Einfluss d. Tetanus d. Muskeln auf d. in ihm enthaltenen Säuren. 399

\section{Versuch V.}

Mittelgrosses Kaninchen. Durchschneidung der Nerven wie vorher. Venöse Blutung die leicht gestillt wurde. 3 Stunden später Tetanus (nur vom Rückenmark aus). Das linke Bein war sehr vollständig gelähmt. Nach $31_{4}$ Stunden starb das Thier ganz unerwartet, die Muskeln waren jedoch fast vollständig erschöpft. Der Leib lag die ganze Nacht in Eis verpackt und war am folgenden Morgen steinhart.

Das Resultat der Verarbeitung war:

a) Links. Lähmung. Fleisch $=135,35 \mathrm{gr}$.

$1 \mathrm{ccm}$ des Aetherauszuges

$=0,46413 \mathrm{ccm}$ derselben NaOH-Lösung.

$$
\begin{aligned}
1 \mathrm{gr} \text { Fleisch } & =0,46413 \times 200 \times \frac{1}{135,35} \\
& =0,6858 \mathrm{ccm} \mathrm{NaOH} \text {-Lösung. } \\
& =0,001306 \mathrm{gr} \mathrm{H}_{2} \mathrm{SO}_{4} .
\end{aligned}
$$

b) Rechts. Tetanus. Fleisch $=128,1 \mathrm{gr}$.

$1 \mathrm{ccm}$ des Aetherauszuges

$$
=0,2298 \mathrm{ccm} \mathrm{NaOH}-\mathrm{Lösung} \text {. }
$$

$1 \mathrm{gr}$ Fleisch $=0,2298 \times 200 \times \frac{1}{128,1}$

$=0,35878 \mathrm{ccm}$ NaOH-Lösung.

$=0,000683 \mathrm{gr} \mathrm{H}_{2} \mathrm{SO}_{4}$.

Vergleicht man diese Zahlen, so erkennt man, dass die Abnahme der Säuren durch keine unzweckmässige Anordnung der Poldrähte bedingt war. Das Verhältniss ist den früheren ähnlich, also :

$$
\begin{gathered}
V^{a} \text { (Lähmung) : } V^{b} \text { (Tetanus) } \\
=1: 0,52 .
\end{gathered}
$$

Dass ich in diesen drei Versuchen die Nerven des linken Schenkels durchschnitt, gesehah nur, weil ich mich bei meinen Präparirïbungen zufällig am linken Schenkel geübt hatte.

Die oben mitgetheilten Untersuchungen lassen alle noch den wichtigen Einwand zu, dass viele der durch den Tetanus abgespaltenen oder veränderten Körper vom Blutstrom weggeschwemmt werden und der Bestimmung entgehen. Hieraus wäre es wohl erklärlich, dass man in dem tetanisirten Muskel weniger Säuren (oder Körper, die bei der hier in Anwendung kommenden Methode Säuren liefern) erhält. Demgemäss schien es wünschenswerth, einen ähnlichen Versuch mit Ausschluss des Blutstroms zu machen. 
Hier sind leider nur Froschschenkel zu gebrauchen. Ieh verfuhr folgendermassen:

Mehrere Frösche wurden geköpft und so zugerichtet, dass nur die Schenkel mit einem längeren Stück der unversehrten Wirbelsäule in Verbindung blieben.

Dann wurde von jedem Paar ein Schenkel im Hüftgelenk abgetrennt und die so gewonnenen Beine, die alle die Haut noch trugen, in Fis verpackt. Das Zurichten geschah überhaupt so, dass die Schenkel auf Eis lagen bis alles zum Tetanisiren bereit war.

Die andern Schenkel mit den daranhängenden Wirbelsäulen wurden so auf Nadeln aufgespiesst, dass der eine Poldraht durch das Rückenmark ging, während der andere sich unten neben den Nerven des plexus sacralis befand. Um den Tetanus gleichmässig zu unterbrechen, schaltete ich ein Metronom nach der bekannten Anordnung ein. Die beiden Versuche wurden im Juni und Juli bei grosser Hitze der Luft angestellt, sodass die Frösche weniger kräftig waren als wünschenswerth gewesen wäre. Die Reizung wurde möglichst lange fortgesetzt. Selbstverständlich fing ich mit den schwächsten wirksamen Strömen an und ging möglichst langsam zu stärkeren über.

Die vollständig erschöpften Muskeln kamen sofort in die Kältemischung, um darin bis zum folgenden Tage zu verweilen.

Die ganze Verarbeitung wurde genau so ausgeführt wie bei den früheren Versuchen mit Kaninchen.

\section{Erster Froschversuch.}

12 mittelgrosse Frösche. Von den zum. Tetanus gebrauchten Schenkeln wurden zwei sehr schwache weggeworfen, da der eine gar nicht arbeitete und der andere starr wurde. Da ich aber bei der raschen Abtrennung der Schenkel etwas tief einschnitt, so erhielt ich von den 10 tetanisirten Schenkeln fast ebensoviel Fleisch als von den 12 andern, die zu diesen Paaren gehörten. Die Muskeln arbeiteten schlecht. Der Versuch dauerte kaum 2 Stunden.

a) Nicht tetanisirte Schenkel. Gewicht des Fleisches $=41,05$ gr. Als Mittel aus 3 gut übereinstimmenden Bestimmungen

$1 \mathrm{ccm}$ des Aetherauszuges $=0,2415 \mathrm{ccm}$ einer sehr verdünnten Natronlange.

$1 \mathrm{ccm} \mathrm{NaOH}$-Lösung $=0,0004303 \mathrm{gr} \mathrm{H}_{2} \mathrm{SO}_{4}$.

$1 \mathrm{gr}$ Fleisch $=0,2415 \times 200 \times \frac{1}{41,05}$

$=1,1766 \mathrm{cem} \mathrm{NaOH}-\mathrm{L}$ ösung

$=0,0005063 \mathrm{gr}^{\mathrm{H}} \mathrm{SO}_{\mathrm{i}}$.

b) Tetanus. Fleisch $=38,0 \mathrm{gr}$.

$1 \mathrm{ccm}$ des Aetherauszuges

$=0,1146 \mathrm{ccm}$ NaOH-Lösung. 
Ueber d. Ein fluss d. Tetanus d. Muskeln auf d. in ihm enthaltenen Säuren. 401

$$
\begin{aligned}
1 \mathrm{gr} \text { Fleisch } & =0,1146 \times 200 \times \frac{1}{38} \\
& =0,60316 \mathrm{ccm} \mathrm{NaOH-Lösung} \\
& =0,00025954 \mathrm{gr} \mathrm{H}_{2} \mathrm{SO}_{4} .
\end{aligned}
$$

Hier hat der Tetanus auch beim Ausschluss des Blutes eine ähnliche Verminderung der sauren Körper erwirkt, wie in den friiheren Versuchen. Das Verhältniss ist

$$
\begin{gathered}
\mathrm{VI}^{\mathrm{a}} \text { (Ruhe) : } \mathrm{VI}^{\mathrm{b}} \text { (Tetanus) } \\
=1: 0,513 .
\end{gathered}
$$

\section{Zweiter Froschversuch.}

In dem vorigen Versuch waren die Schenkel näch Belieben rechts oder links abgeschnitten worden. Bei einem Controllversuch nahm ich die rechten $\mathrm{ab}$ und benutzte die linken zum Tetanus. Es wurden 13 Frösche einer neuen Sendung gebraucht, die alle grösser und etwas kräftiger waren wie die im ersten Froschversuch. Die Dauer des Tetanisirens betrug nicht ganz $3^{1 / 4}$ Stunden.

a) Nicht tetanisirte Schenkel. Gewicht des Fleisches $=65,3 \mathrm{gr}$.

$1 \mathrm{ccm}$ des Aetherauszuges

$=0,040167 \mathrm{ccm}$ einer andern $\mathrm{NaOH}-\mathrm{Lösung}$.

$1 \mathrm{ccm} \mathrm{NaOH}-$ Lösung $=0,003942 \mathrm{gr} \mathrm{H}_{2} \mathrm{SO}_{4}$.

$1 \mathrm{gr}$ Fleisch $=0,040167 \times 200 \times \frac{1}{65,3}$

$$
\begin{aligned}
& =0,123023 \mathrm{ccm} \mathrm{NaOH}-L o ̈ s u n g . \\
& =0,000485 \mathrm{gr} \mathrm{H} \mathrm{H}_{2} \mathrm{SO}_{4} .
\end{aligned}
$$

b) Tetanus. Fleisch $=60,95 \mathrm{gr}$.

$1 \mathrm{ccm}$ des Aetheranszuges

$=0,0224 \mathrm{ccm}$ dieser $\mathrm{NaOH}$-Lösung.

$$
\begin{aligned}
1 \text { gr Fleisch } & =0,0224 \times 200 \times \frac{1}{60,95} \\
& =0,0734 \mathrm{ccm} \mathrm{NaOH}-\mathrm{Lösung} \\
& =0,000289 \mathrm{gr} \mathrm{H}_{2} \mathrm{SO}_{4} .
\end{aligned}
$$

Das Verhältniss dieser Zahlen ist dem der früheren Versuche ganz ähnlich, nämlich:

$\mathrm{VII}^{\mathrm{a}}$ (Ruhe) $:$ VII $^{\mathrm{b}}$ (Tetanus) $=1: 0,596$

Zur Uebersicht lassen sich die Resultate dieser sieben Versuche in einer kleinen Tabelle zusammenstellen. 


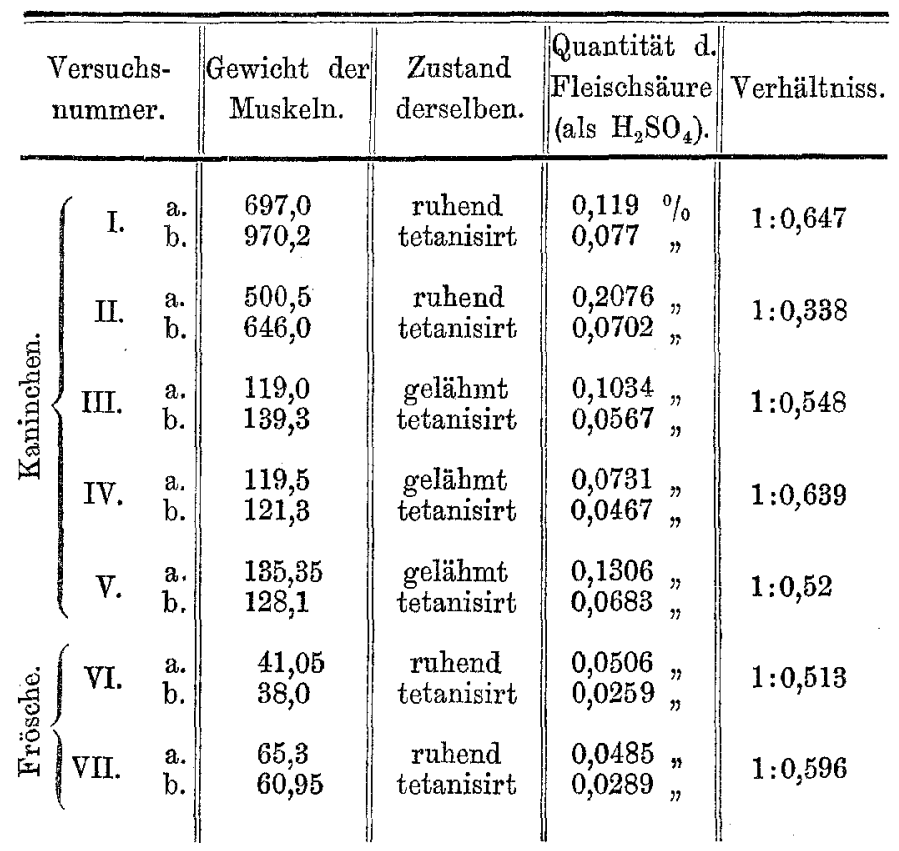

Versuch II dieser Tabelle wird nur deshalb mitgetheilt, weil er trotz des bedeutenden Fehlers in demselben Sinn ausfällt, wie die übrigen.

Es verdient noch hervorgehoben zu werden, dass nur die hier beschriebenen Versuche angestellt wurden, so dass die Abnahme der Säuren in jedem Versuch um so bemerkenswerther ist, weil keine Ausnahme constatirt wurde.

Man könnte daran denken, dass die verschwundenen Säuren sich in dem abdestillirten Aether finden liessen. Auch dieses Verhalten habe ich in einigen Fällen geprïft, ohne für diesen Einwand irgend welche Stütze zu finden. Ich mass das Volum des wieder gewonnenen Aethers (die Reste bei der Destillation waren ziemlich gleich gross gewesen), uibersättigte $100 \mathrm{ccm}$ mit Natronlauge and titrirte mit einer Salzsäurelösung zurtick. Diese Zahlen auf das Ganze berechnet und dann auf ein Gramm Fleisch bezogen ergaben :

IV Lähmung. Auf $1 \mathrm{gr}$ Fleisch kommt ein Säuregehalt = $0,0782 \mathrm{ccm}$ einer sehr verdünnten Salzsäurelösung.

IV $^{b}$ Tetanus. Auf $1 \mathrm{gr}$ Fleisch 0,044 cem HCl-Lösung.

$$
\begin{aligned}
\mathrm{IV}^{\mathrm{a}}: \mathrm{IV}^{\mathrm{b}} & =0,0782: 0,044 . \\
& =1: 0,563 .
\end{aligned}
$$


Ueber d. Einfluss d. Tetanus d. Muskeln auf d. in ihm enthaltenen Säuren. 403

$V^{\text {a }}$ Lähmung. Auf $1 \mathrm{gr}$ Fleisch $0,0183 \mathrm{ccm}$ HCl-Lösung.

$\mathrm{V}^{\mathrm{b}}$ Tetanus. Auf $1 \mathrm{gr}$ Fleisch 0,0137 ccm HCl-Lösung.

$$
\begin{aligned}
\mathrm{V}^{\mathrm{a}}: \mathrm{V}^{\mathrm{b}} & =0,0183: 0,0137 . \\
& =1: 0,748 .
\end{aligned}
$$

Bei den beiden Froschversuchen habe ich eine ähnliche Prüfung unternommen. In dem ersten glanbe ich ebenfalls eine Abnahme der flüehtigen Sänren constatirt zu haben. Das Verhältniss war etwa 1:0,87, es waren jedoch die Quantitäten so klein und die Bestimmung dieser Säuren hier sowie im zweiten Versuch so unsicher, dass ich darauf verzichten musste, brauchbare Zahlen zu erhalten,

Als ich diese Versuche nunmehr zum Abschluss gebracht hatte, wurden wir auf die neuen Resultate Boehm's betreffs der Schwierigkeit der vollständigen Gewinnung des Muskelglykogens aufmerksam. Es schien wünschenswerth auch unsre Methode (die den bisher gebräuchlichen Methoden zur Bestimmung der Milchsäure ähnlich ist) einer neuen Prüfung zu unterziehen, um nämlich das Verhältniss der Auszüge mittelst eines verdünnteren Alkohols kennen zu lernen. $\mathrm{Zu}$ diesem Ende wurde ein neuer Versuch gemacht. Alle Cautelen wurden genau beobachtet, um jeden Verlust zu verhindern, namentlich wurden die Niederschläge mit grösserer Sorgfalt ausgewaschen resp. ausgekocht, un auch hier keinen Verlust an den gesuchten Substanzen zu erleiden.

\section{Versuch VIII.}

Durchschneidung der Nerven eines mittelgrossen Kaninchens wie in Versuchen III-V. Etwa 4 Stunden später Tetanisirung des intakten Schenkels vom Rückenmark aus. Nach $3 \frac{1}{2}$ Stunden plötzlicher Tod des Thieres durch Verblutung in die Wunde des linken Schenkels; sofortige Verpackung in Eis. Am nächsten Morgen wurde das steinharte. Fleisch abgetrennt, mit der Wurstmaschine zerkleinert, gewogen und in Alkohol $(87 \%)$ gebracht. Nachdem dieser Muskelbrei in einer Reibschale mit dem Alkohol gut gerieben war, wurde von letzterem so viel zugesetzt, dass etwa $500 \mathrm{ccm}$ in Anwendung kamen. Das Ausziehen geschah in einem mässig warmen Zimmer und wurde durch häufiges Umrïhren beschleunigt. Nach 24 Stunden wurde der Alkohol abgegossen, die Fleischmasse sorgfältig ausgedrückt, nochmals mit frischem Alkohol gerieben und zu weiterem Ausziehen hingestellt. - Diese Behandlung wurde so lange fortgesetzt bis der Alkohol des sechsten Aus* zuges keine wägbaren Substanzen aus dem Fleisch aufgenommen zu haben schien. Um die Resultate mit denen früherer Versuch vergleichbar zu machen, 
wurden die sechs Auszïge paarweise (also I-II, u. s. w. zusammen) verarbeitet. Dies geschah wie bei den andern Versuchen.

Die Titrirung der Aetherauszüge wurde mit einer sehr verdünnten Aetzbarytlösung ausgeführt, von der $1 \mathrm{ccm}=0,0011212 \mathrm{gr} \mathrm{H}_{2} \mathrm{SO}_{4}$ war.

Die Ergebnisse dieser Titration sind:
a) Links. Lähmung.
b) Rechts. Tetanus.

Fleisch $=80,5 \mathrm{gr}$.

Fleisch $=78,1 \mathrm{gr}$.

1 gr Fleisch nach den Auszügen

$$
\begin{aligned}
& \text { I一II. }=0,00822 \quad \mathrm{gr} \mathrm{H}_{2} \mathrm{SO}_{4}=0,00256 \quad \mathrm{gr} \mathrm{H} \mathrm{H}_{2} \mathrm{SO}_{4} \\
& \text { III-IV. }=0,00155, \quad=0,00114 \pi \text { " } \\
& \text { V-VI. }=0,000156 \%, \quad=0,000275 \%, \\
& \text { Zusammen }=0,00493 \text { gr } \mathrm{H}_{2} \mathrm{SO}_{4}=0,00397 \mathrm{gr} \mathrm{H}_{2} \mathrm{SO}_{4}
\end{aligned}
$$

Fasst man das Verhältniss dieser Zahlen ins Auge, so verhält sich beim

I-II. Auszuge $a: b=1: 0,79$

$$
\begin{array}{rll}
\text { III-IV. } & \quad & \quad=1: 0,73 \\
\text { V-VI. } & \quad & \quad=1: 1,76(! ?)
\end{array}
$$

oder zusammen

$$
\begin{gathered}
\text { VIII (Lähmung) : VIIb (Tetanus) } \\
=1: 0,805 .
\end{gathered}
$$

Hieraus geht nun deutlich hervor, dass das Ausziehen mit absolutem Alkohol bei den früheren Versuchen kein vollständiges gewesen ist, ohne dass jedoch dadurch das Verhältniss der gewonnenen Substanzen bedeutend beeinflusst worden wäre. Dass Astaschewsky trotz länger dauerndem, dreimal wiederholtem Ausziehen Zahlen erhielt, die mit meinen frïheren sehr gut übereinstimmen, mag auch (neben andern möglichen Verlusten, die seine Methode mit sich bringt), darin seinen Grund haben, dass er ebenfalls mit absolutem Alkohol arbeitete. Merkwürdig ist freilich das Verhältniss der Säuren in den dritten Portionen (Auszügen V-VI) obigen Versuches, wenn nicht möglicherweise eine Verwechselung stattgefunden hat. Jedenfalls ist diese auffallende Aenderung ohne besonderen Einfluss auf das Gesammtverhältniss.

Leider fehlt es mir vorläufig an Zeit, diese interessanten aber sehr zeitraubenden Versuche zu wiederholen, um die neuen Zahlen zu prüfen, sowie um das Verhalten der Salze zu studiren. Ich glaube jedoch, dass die Titrirung der mit Anwendung der nöthigen Cautelen gewonnenen Aetherauszuige mindestens ebenso befriedigend ausfallen dürfte wie die übliche Bestimmung der Salze, die allenfalls nur mit bedeutenden Verlusten auszufuihren ist. 
Ueber d. Einfluss d. Tetanus d. Muskeln auf d. in ihm enthaltenen Säuren. 405

\section{Resultat.}

Herr Professor Pflüg e r hat mir nun folgende Betrachtung mitgetheilt:

Diese Untersuchungen haben also zu dem auffallenden Ergebniss geführt, dass durch den Tetanus aie Zahl der sauren (in Aether löslichen) Molecüle vermindert wird. Da ich meine Experimente vielfach sehr variirt habe und stets mit grösster Sorgfalt ein gleichmässiges analytisches Verfahren bei den entsprechenden Versuchen einzuhalten bestrebt war und immer wieder ausnahmslos dieselbe bedeutende Abnahme der Zahl der sauren Molecüle constatirte, so kann ich an der Richtigkeit dieses Ergebnisses nicht zweifeln.

Es fragt sich also, wie die Paradoxie zu verstehen ist, dass die Muskeln durch den Tetanus sauer werden, durch in Aether lösliche Säuren (Milchsäure), obwohl die Zahl der sauren in Aether löslichen Moleciile abnimmt.

Hier liegt es nahe, daran zu denken, dass die Milchsäuren zu Condensationen sehr geneigt sind, indem z. B. durch Einwirkung des Carboxyls in einem Milchsäuremolecül auf das alkoholische Hydroxyl in einem anderen Milchsäuremoleciul unter Austritt von Wasser Aetherbildung stattfindet: z. B.

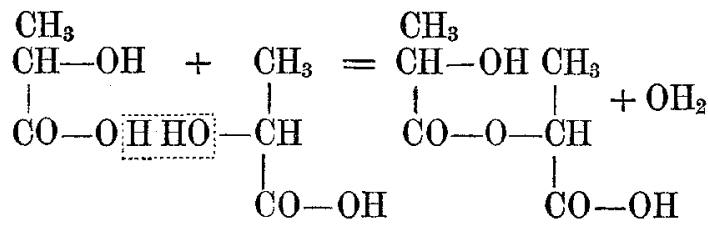

2 Molecïle Milchsäure. Condensirte Milchsäure.

Nachdem diese Condensation oder Aetherbildung stattgefunden hat, ist die Zahl der Carboxyle von 2 auf 1 vermindert: die Acidität hat abgenommen: die condensirte Säure sättigt nur halb so viel Alkali zu neutralem Salz.

Umgekehrt ist es bekannt, dass die neutral reagirenden Salze dieser condensirten Milchsätren in hohem Grade zersetzbar sind, indem sie unter Wasseraufnahme sich wieder in ihre Constituenten spalten, so dass die vorher neutrale Flüssigkeit wieder sauer reagirt. 
Kommen nun im Muskel solche condensirte Milchsäuren vor, so kann die ursprünglich neutrale Reaction allmählig intensiv sauer werden, ohne dass ein Molecül neuer Säure zu entstehen braucht. Die Titration misst deshalb nicht die wägbare Menge der Säuren, sondern wie wir uns ausdriickten, nur die Zahl der sauren Molecüle. So erklären sich die Thatsachen durch die Annahme, dass durch den Tetanus eine Spaltung condensirter in einfachere Molecüle veranlasst wird. Da dies eine Vermehrung der sauren Moleciile bedingt, deren Gesammtsumme gleiehwohl durch den Tetanus abnimmt, so wird es allerdings in hohem Grade wahrscheinlich, dass jene Vermehrung der Zahl - welche die saure Reaction bedingt - übercompensirt wird durch einen vom Tetanus herrïhrenden Verbrauch der Molecille, resp. Oxydation zu Kohlensäure und Wasser.

Zum Schlusse sage ich Herrn Geheimrath Pflüger für seine freundliche Unterstützung und Leitung bei dieser kleinen Arbeit herzlichsten Dank.

(Physiologisches Laboratorium in Bonn.)

\section{Weitere Beiträge zur quantitativen Analyse der Chloride in salpetersaurer Harnbarytmischung.}

Von

\section{Dr. Louis Habel,}

I. Assistenten am physiologischen Laboratorium.

Aus den im XXIII. Bande dieses Archivs von mir und J. Fernholz publicirten Versuchen, worauf wir eine Methode der Bestimmung der Chloride direct in der Harnbarytmischung grüdeten, ging hervor, dass die durch salpetersaures silberoxyd in salpetersaurer Lösung fällbaren Verbindungen im normalen mensch- 